\title{
EL TRABAJO DE CAMPO EXPERIMENTAL COMO MÉTODO DE APRENDIZAJE PRÁCTICO
}

\author{
Nicolás Fernández Losa \\ Carlos González Menorca \\ Francisco Javier Ruiz Cabestre \\ Universidad de La Rioja
}

\begin{abstract}
RESUMEN: Este trabajo describe una experiencia docente sobre el trabajo de campo experimental como método de aprendizaje práctico implantada en la asignatura de Comportamiento Organizativo. Con esta experiencia docente se pretende modificar la formación práctica y sus procesos evaluativos, con el objeto de favorecer el desarrollo de las competencias transversales del alumnado. Para ello, la utilización de un plan de prácticas, abordado a través de un trabajo de campo experimental y llevado a cabo con la colaboración de una organización empresarial en el seno de un equipo de trabajo (como unidad orgánica de aprendizaje), surge como una alternativa al método tradicional de enseñanzas prácticas y permite el acercamiento de la realidad empresarial al aula, así como fomentar de forma activa el empleo de habilidades transversales. En concreto, la experiencia se desarrolla en tres fases sucesivas. Inicialmente, los alumnos, tras formar un grupo de trabajo y definir un proyecto de trabajo de campo, deben conseguir la colaboración de una organización empresarial del entorno en la que obtener datos sobre una o varias áreas funcionales de su comportamiento organizacional. Posteriormente, los alumnos Ilevan a cabo el trabajo de campo con la realización de las visitas programadas y elaboran una memoria en la que establecen un diagnóstico sobre la estrategia seguida por la empresa en dichas áreas funcionales, al objeto de proponer y justificar alternativas de actuación que mejoren las existentes. Finalmente, los profesores evalúan las diferentes memorias de los trabajos de campo y sus exposiciones públicas de acuerdo con unas rúbricas de evaluación, que tratan de objetivar y unificar al máximo los criterios de evaluación y sirven de guía del proceso de aprendizaje de los alumnos. Los resultados de implantación de esta experiencia docente, medidos a través de un cuestionario Likert, son muy satisfactorios para los alumnos.
\end{abstract}


PALABRAS CLAVE: Innovación docente, formación práctica, trabajos de campo experimentales, equipos de trabajo, habilidades transversales, sistemas de evaluación.

\title{
THE EXPERIMENTAL FIELD WORK AS PRACTICAL LEARNING METHOD
}

\begin{abstract}
This paper describes a teaching experience about experimental field work as practical learning method implemented in the subject of Organizational Behaviour. With this teaching experience we pretend to change the practical training, as well as in its evaluation process, in order to favour the development of transversal skills of students. For this purpose, the use of a practice plan, tackled through an experimental field work and carried out with the collaboration of a business organization within a work team (as organic unity of learning), arises as an alternative to the traditional method of practical teachings and allows the approach of business reality into the classroom, as well as actively promote the use of transversal skills. In particular, we develop the experience in three phases. Initially, the students, after forming a working group and define a field work project, should get the collaboration of a nearby business organization in which to obtain data on one or more functional areas of organizational behaviour. Subsequently, students carry out the field work with the realization of the scheduled visits and elaboration of a memory to establish a diagnosis of the strategy followed by the company in these functional areas in order to propose and justify alternative actions that improve existing ones. Finally, teachers assess the different field work memories and their public presentations according to evaluation rubrics, which try to objectify and unify to the maximum the evaluation criteria and serve to guide the learning process of students. The results of implementation of this teaching experience, measured through a Likert questionnaire, are very satisfactory for students.
\end{abstract}

KEYWORDS: Teaching innovation, practical training, experimental field works, work teams, transversal skills, evaluation systems.

Recibido: 25/03/2014

Aceptado: 08/11/2014

\section{INTRODUCCIÓN}

El Espacio Europeo de Educación Superior (EEES) y el sistema de transferencia y acumulación de créditos (ECTS) representan un cambio sin precedentes en la formación superior universitaria, que requiere una reconceptualización de la cultura docente universitaria (Palomares, 2011). Para ello, es necesario plantear nuevas estrategias de enseñanza-aprendizaje y sistemas de evaluación, donde el aprendizaje del alumno sea el centro de atención sobre el que pivote el nuevo modelo pedagógico universitario. De esta forma, y frente al modelo pedagógico tradicional, que se concreta básicamente en la transmisión unidireccional de conocimientos desde el profesor al alumnado, el nuevo modelo pedagógico que se plantea con la 
Convergencia Europea estará focalizado en el estudiante y en su autonomía a la hora de gestionar los conocimientos a adquirir (Sayago et al., 2011), dado que el estudiante deberá abandonar su rol pasivo de recepción y asimilación de conocimientos en favor de un rol más activo donde necesariamente deberá buscar y elaborar dichos conocimientos. Además, este nuevo modelo pedagógico va más allá de la mera adquisición de conocimientos y se dirige a la formación en competencias. Esto es, a la adquisición de conocimientos hay que sumarle las actitudes y las habilidades o destrezas necesarias que el desempeño del ejercicio profesional exigirá a los futuros egresados universitarios ${ }^{1}$.

En suma, la puesta en marcha del nuevo marco educativo supone centrar el proceso de aprendizaje no solo sobre los contenidos sino también sobre las competencias, tanto transversales como específicas, y hacer del alumnado el protagonista de dicho proceso (Ion y Cano, 2012). Asimismo, el profesor debe ceder terreno en favor del propio estudiante, para favorecer una mayor autonomía e independencia en su aprendizaje (Jenaro et al., 2013), y poner en práctica nuevas forma de enseñanza-aprendizaje y sistemas de evaluación que propicien la formación en competencias del alumnado (Laffon y Pasáro, 2011; Béjar y Merino, 2012).

En este contexto se presenta la actual experiencia docente, que pretende cambiar el diseño y la aplicación de la formación práctica, así como sus procesos evaluativos, con el objeto de favorecer el desarrollo de las competencias transversales del alumnado². Para ello, la utilización de un plan de prácticas, abordado a través de un trabajo de campo experimental con la colaboración de una organización empresarial en el seno de un equipo de trabajo (como unidad orgánica de aprendizaje), surge como una alternativa al método tradicional de enseñanzas prácticas. Además, este cambio permite acercar la realidad empresarial al aula, mediante la puesta en práctica de las competencias específicas, así como fomentar de forma activa el empleo de competencias transversales entre el alumnado.

Esta experiencia docente se desarrolla en tres fases sucesivas. En un primer momento, los alumnos deciden su participación en un grupo de trabajo operativo y, tras definir un proyecto de trabajo de campo, deben conseguir la colaboración de una organización empresarial del entorno en la que obtener datos sobre una o varias áreas funcionales de su comportamiento organizacional. Posteriormente, los alumnos llevan a cabo el trabajo de campo con la realización de las visitas programadas y, tras la obtención de los datos necesarios, elaboran una memoria en la que establecen un diagnóstico sobre la estrategia seguida por la empresa en dichas áreas fun-

1. Nótese que las competencias se pueden agrupar en transversales, también denominadas genéricas, y específicas, también conocidas como profesionales. Las primeras hacen referencia a un conjunto de conocimientos, actitudes y habilidades o destrezas comunes para todas las áreas del saber, mientras que las segundas son particulares de cada carrera profesional (Gutiérrez y De Pablos, 2010).

2. La adaptación de los métodos de enseñanza-aprendizaje y de los sistemas de evaluación al Espacio de Convergencia Europeo ha motivado la puesta en marcha de numerosas experiencias docentes, entre las que, a título de ejemplo, cabe señalar Sayago et al. (2011), Palomares (2011), Astoreca (2012) y Flórez y Albelda (2012). 
cionales, al objeto de proponer y justificar alternativas de actuación que mejoren las existentes. Finalmente, los profesores evalúan las diferentes memorias de los trabajos de campo y sus exposiciones públicas, siguiendo una metodología de rúbricas de evaluación, diseñadas para dicho cometido, que trata de objetivar y unificar al máximo los criterios de evaluación y de servir de guía del proceso de aprendizaje de los alumnos (García-Ros, 2011; García-Ros et al., 2012). Por otro lado, la modificación de los sistemas de evaluación, atendiendo a las rúbricas de los criterios de evaluación, fomenta la puesta en práctica de las competencias transversales por parte de los alumnos.

La aplicación de esta experiencia en la asignatura Comportamiento Organizativo (asignatura preliminar a la de Dirección de Recursos Humanos), impartida en los Grados de Administración y Dirección de Empresas, Derecho, Trabajo Social, y Relaciones Laborales y Recursos Humanos, Ileva consigo que la formación práctica, realizada normalmente en clases prácticas tradicionales de 25 a 30 alumnos, se convierta en un trabajo de campo experimental, abordado en organizaciones empresariales del entorno y en el seno de un de equipo de trabajo. Asimismo, este cambio, además de poner en práctica las competencias específicas propias de la asignatura, adquiridas durante las clases teóricas, fomenta de forma activa el empleo de competencias transversales.

En resumen, a través de esta nueva experiencia se intentan poner en práctica mejoras substanciales en dos ámbitos del aprendizaje práctico: el relativo al proceso de enseñanza-aprendizaje y el referido al sistema de evaluación. Atendiendo al proceso de enseñanza-aprendizaje, los cambios están enfocados a aproximar la práctica de la asignatura Comportamiento Organizativo a la realidad empresarial mediante la aplicación de competencias transversales por parte de los alumnos. Esto es, se trata de conocer in situ la problemática empresarial y, para ello, se motiva el trabajo autónomo, se practica la cultura del trabajo en equipo mediante actitudes colaborativas, se propicia el aprendizaje autónomo, reflexivo y crítico, se fomenta la comunicación escrita y oral, y se incentiva la utilización de las nuevas Tecnologías de la Información y Comunicación (TICs). En cuanto a los cambios en el sistema de evaluación, cabe indicar que tratan de buscar la unificación y objetivación de los criterios de evaluación, tanto de las memorias elaboradas por los grupos de trabajo como de sus exposiciones públicas, y están diseñados con fines formativos para los alumnos, dado que sirven de guía para la preparación y elaboración de ambas tareas (esto es, la memoria y la exposición pública).

En lo que resta el trabajo se estructura en las siguientes secciones: la segunda describe la experiencia docente; la tercera presenta algunos aspectos más concretos de la aplicación de la experiencia docente (como son las labores de supervisión, preparación del cuestionario y de la entrevista, y elaboración de la memoria del trabajo de campo); la cuarta muestra la evaluación de los trabajos y de las presentaciones públicas a partir de las rúbricas de evaluación; la quinta recoge la valoración que los alumnos otorgan a dicha experiencia docente, tanto en lo relativo a su aplicación como en lo concerniente a las competencias trasversales adquiridas, y la sexta sintetiza las conclusiones más relevantes obtenidas. 


\section{DesCRIPCIÓN DE LA EXPERIENCIA DOCENTE}

El desarrollo de esta experiencia de innovación docente se ha llevado a cabo durante el curso académico 2011/12 por un equipo de profesores del Departamento de Economía y Empresas de la Universidad de La Rioja (UR) dentro del marco de la asignatura básica de Comportamiento Organizativo (que ha contado con 334 alumnos matriculados), impartida en el primer curso de los Grados de Administración y Dirección de Empresas (que representa el 52,69\% de los alumnos), Derecho (el $18,56 \%$ ), Trabajo Social (el $19,76 \%$ ) y Relaciones Laborales y Recursos Humanos (el 8,98\%). Estos alumnos son mayoritariamente recién incorporados a la universidad (el 39,22\% tiene 18 años, el 18,86\% 19, el 12,87\% 20 y el resto, el 29,04\%, tiene más de 20 años), predominan las mujeres (con el 58,38\% y, especialmente, en el Grado de Trabajo Social, con el $83,33 \%$ ) y los repetidores representan un número muy bajo (el $11,08 \%$ ).

Para ello, la formación práctica obligatoria, tradicionalmente encaminada a la consecución de las competencias específicas o profesionales, que habitualmente se realiza en clases prácticas tradicionales de 25 a 30 alumnos, ha sido reemplazada por un trabajo de campo experimental, que se ha abordado en una organización empresarial del entorno más inmediato mediante la constitución y desarrollo de un equipo de trabajo, con el objeto de alcanzar las siguientes competencias transversales o genéricas:

- Implicar y motivar a los alumnos en el desarrollo práctico de la asignatura.

- Favorecer el trabajo autónomo del alumno y, por tanto, su autoaprendizaje.

- Poner en práctica actitudes colaborativas entre alumnos y la cultura del trabajo en equipo.

- Conseguir que el alumno se aproxime a la realidad de las competencias profesionales que la sociedad, en su conjunto, y el mundo empresarial, en particular, le van a exigir.

- Propiciar la reflexión y valoración crítica del alumno.

- Enriquecer la comunicación escrita del alumno (elaboración de la memoria).

- Desarrollar la comunicación oral del alumno (exposición pública de la memoria).

- Contribuir a la utilización de las nuevas Tecnologías de la Información y Comunicación (TICs).

Además, se ha diseñado un sistema de evaluación acorde con estas competencias. Para ello, como se verá más adelante, se ha establecido un sistema de rúbricas de los criterios de evaluación que intenta, en la medida de lo posible, unificar y objetivar el proceso de evaluación, tanto por parte de los profesores (respecto de la memoria y exposición pública) como por parte de los alumnos (en relación con la exposición pública), y servir de guía a los alumnos para la preparación y elaboración de la memoria y de su exposición pública.

Para la consecución de estas competencia transversales se han puesto en marcha una serie de actividades estructuradas en el espacio y en el tiempo en tres fases, tal y como muestra la Figura 1. 

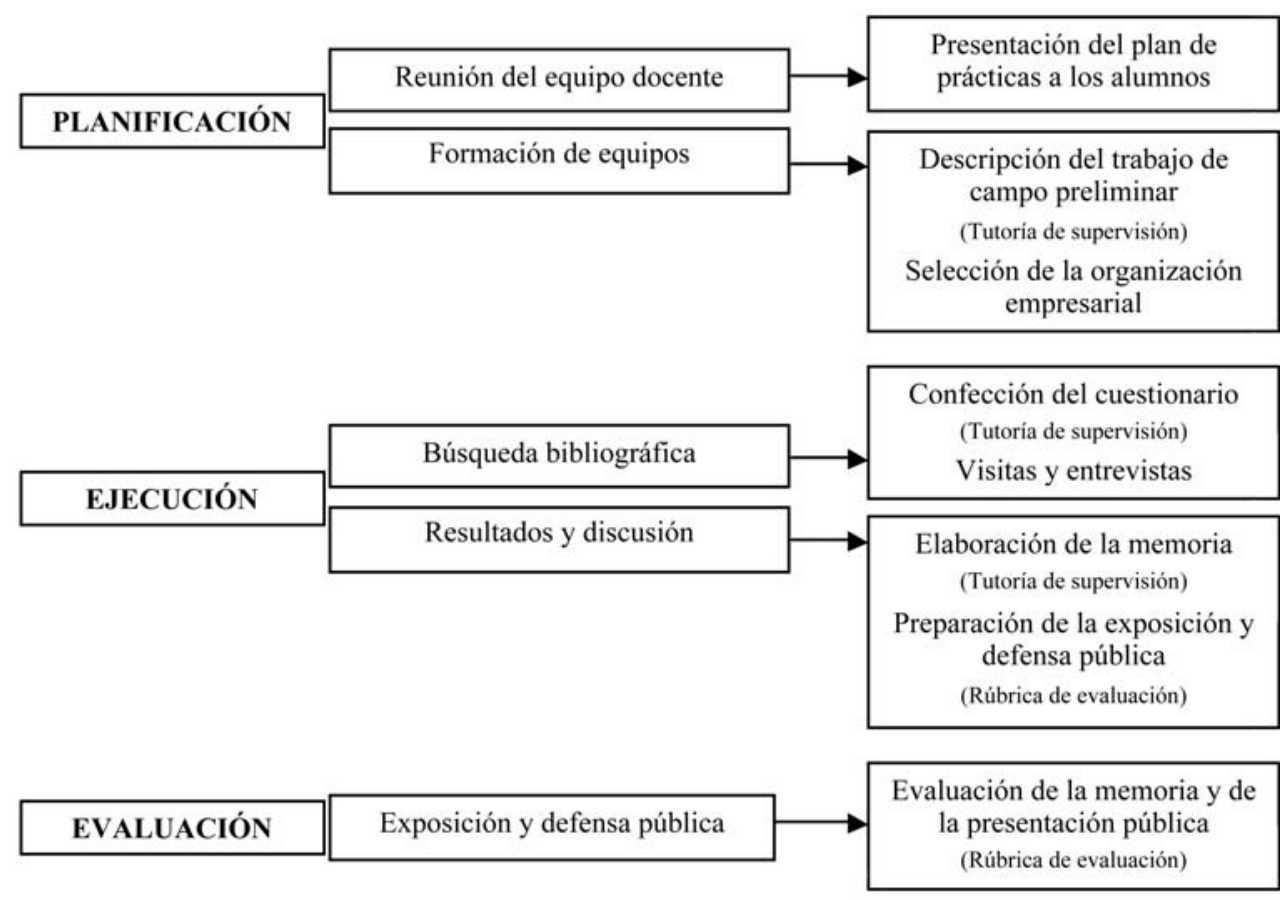

Figura 1. Esquema de la experiencia docente

Estas actividades son:

- Planificación: los profesores elaboran un plan de prácticas que presentan a los alumnos y estos últimos deciden su participación en un grupo de trabajo operativo (de tres o cuatro personas) que deberán autorregularse y autogestionarse. Este plan de prácticas consistirá en la elaboración de un trabajo de campo experimental con la colaboración de una organización empresarial en el seno de un equipo de trabajo. Para ello, en una sesión de tutoría, cada equipo presentará el proyecto del trabajo de campo que desea llevar a cabo y discute los pormenores con los profesores. Posteriormente, cada equipo debe conseguir la colaboración de una empresa del entorno en la que obtener datos sobre una o varias áreas funcionales de su comportamiento organizacional.

- Ejecución: el equipo formado, tras realizar una búsqueda y selección bibliográfica acorde con los objetivos planteados en su proyecto de trabajo de campo, debe elaborar un cuestionario con las preguntas que formularán a las personas contactadas de la organización empresarial seleccionada. En una segunda sesión de tutoría, cada equipo revisa con los profesores el cuestionario de la entrevista a realizar y, posteriormente, en una o dos visitas programadas previamente, realiza las entrevistas para la obtención de datos relevantes. A continuación, los alumnos elaboran una memoria en la que establecen un diagnóstico sobre la estrategia seguida por la empresa en el ámbito de la gestión de los recursos humanos al objeto de proponer y justificar alternativas de 
actuación que mejoren las existentes. Finalmente, antes de exponer en clase ante sus compañeros el trabajo realizado, en una tercera sesión de tutoría, cada equipo revisa con los profesores la memoria que ha confeccionado. Estas tareas relativas a la elaboración de la memoria y a la preparación de la exposición pública contarán con el apoyo de las rúbricas de evaluación, que ejercen una labor formativa (Martínez-Figueira et al., 2013; Panadero y Jonsson, 2013; Gallego y Raposo-Rivas, 2014).

- Evaluación: cada equipo lleva a cabo la presentación de los resultados de su trabajo ante los profesores y compañeros, utilizando las Tecnologías de la Información y la Comunicación (TICs) oportunas, evaluando además las fortalezas y debilidades que hayan podido percibir en su funcionamiento como equipo de trabajo. Finalmente, los profesores evaluarán la memoria del trabajo de campo y su presentación pública, y el resto de compañeros también emitirán su opinión sobre la exposición pública, basándose ambos en los criterios de evaluación previstos por las rúbricas propuestas.

\section{AlgunOS ASPECTOS PRÁCtICOS DE APLICACIÓN DE LA EXPERIENCIA DOCENTE: TUTORÍAS DE SUPERVISIÓN, PREPARACIÓN DEL CUESTIONARIO Y DE LA ENTREVISTA, Y ELABORACIÓN DE LA MEMORIA DEL TRABAJO DE CAMPO}

\subsection{Tutorías de supervisión}

En el esquema de la Figura 1 se hace referencia a tres tutorías de supervisión que se realizan en distintos momentos de la experiencia docente descrita. En la primera de las tutorías se tiene una reunión con el equipo recién formado para analizar conjuntamente, profesores y alumnos, el proyecto de trabajo de campo que quieren llevar a cabo: el sector laboral que les interesa conocer, la organización empresarial concreta que desean analizar, las habilidades directivas que más les motiva explorar, etc.

La segunda tutoría se realiza para discutir el cuestionario elaborado con motivo de la entrevista, que se llevará a cabo a los profesionales relevantes de la organización empresarial en materia de comportamiento organizacional (normalmente, el director o responsable de recursos humanos o, en su caso, el gerente).

Por último, la tercera de las tutorías tiene lugar cuando la elaboración de la memoria del trabajo de campo está bastante avanzada, con el objetivo de valorar los datos obtenidos y perfilar la presentación que se realizará ante los profesores y compañeros de clase.

\subsection{Preparación del cuestionario y de la entrevista}

Los alumnos preparan las preguntas específicas que constituyen el cuestionario, que versará sobre temas propios del comportamiento organizacional. En concreto, sobre el departamento de recursos humanos y su funcionamiento; el trabajo en equipo; la captación, selección e integración del personal; la motivación, retribución e incentivos; el estilo de dirección y liderazgo; la comunicación interpersonal y organizativa; la política de formación en la empresa; las nuevas exigencias de la gestión internacional de los recursos humanos; la gestión de la seguridad y la salud en el tra- 
bajo; la gestión de las relaciones con los representantes de los trabajadores; el respeto de los derechos de los empleados y gestión de la disciplina; el diseño y administración de las prestaciones sociales; la evaluación y retribución del desempeño; el desarrollo de la carrera profesional; la gestión del despido de trabajadores, reducción del tamaño empresarial y colocación externa; la igualdad de oportunidades; la gestión de la diversidad de la plantilla, etc. Este cuestionario será discutido en la segunda tutoría de supervisión antes de llevarse a cabo la entrevista.

Para la realización de las entrevistas se les indica a los alumnos las siguientes pautas de comportamiento:

- Presentarse a la persona a entrevistar y hablarle brevemente acerca del proyecto a desarrollar, sobre todo de cómo se va a tratar la información y de cómo la empresa va a estar informada de lo que se escriba.

- Antes de iniciar la entrevista conviene detallar los pormenores de la misma: tipo de preguntas a formular, duración, posibilidad de grabación, etc.

- A continuación se inicia la entrevista, preguntándole su nombre y cargo, y todas aquellas preguntas generales que aporten información rica en anécdotas y matices. Entre las que cabe señalar: ¿A qué se dedica su organización? ¿Nos podría contar la historia de su empresa? ¿Cuál fue el germen? ¿Cuáles han sido los éxitos y fracasos más relevantes que ha vivido en su organización? ¿Cuáles son los principales cambios que ha experimentado su organización? ¿Por qué cambió? ¿Cómo? ¿Quiénes son los principales protagonistas de la historia de la empresa? ¿Cuáles cree que han sido los factores clave del éxito de su organización? ¿Cómo ve el futuro?

- Posteriormente se formularán las preguntas específicas que constituye el cuestionario y que previamente ha sido elaborado y revisado.

- En la entrevista se recomienda intentar identificar a otros actores clave que pueda resultar interesante entrevistar por su protagonismo.

- Antes de cerrar la entrevista conviene preguntar al entrevistado si quiere aportar alguna información adicional. También es interesante preguntarle por su predisposición a seguir colaborando, si fuera necesaria información adicional, y, por supuesto, agradecerle la atención prestada.

- Por último se solicitará de la empresa todo tipo de información pública disponible: folletos, revistas, páginas web, etc.

\subsection{Elaboración de la memoria del trabajo de campo}

Para elaborar la memoria del trabajo de campo se proporciona el siguiente esquema que sirve de guía a los alumnos:

- Portada: contendrá el título, el logotipo relativo a la organización empresarial que se va a analizar, los miembros que componen el grupo de trabajo, la asignatura, el curso académico, etc.

- Índice: con los apartados correspondientes y el número de página.

- Contenido: como mínimo contendrá los siguientes epígrafes: 
1. Presentación de la empresa: se mostrará su historia, su evolución, y los hechos más destacables, así como los datos más relevantes de la empresa: producción, ventas, número de trabajadores y otros datos de interés.

2. Objetivos de la organización empresarial y del departamento de recursos humanos: se definirán dichos objetivos, así como los conceptos de misión, visión y valores, tanto para la empresa como para el departamento de recursos humanos.

3. Función del departamento de recursos humanos: se realizará un análisis de los integrantes del departamento de recursos humanos (miembros que lo forman, perfil académico de los mismos, tareas o responsabilidades atribuidas, etc.) y un análisis de toda la plantilla de la empresa (nivel de estudios, número de trabajadores, edad media, distribución por sexo, etc.).

4. Organigrama: se definirá el organigrama actual y los cambios más recientes del pasado.

5. Políticas de personal: se describirá cómo se han gestionado las principales áreas funcionales del departamento de recursos humanos. En particular, su funcionamiento, la captación y selección del personal, el sistema de retribución e incentivos, el estilo de dirección y liderazgo, la comunicación interpersonal y organizativa, la política de formación en la empresa, la seguridad en el trabajo, las relaciones con los representantes de los trabajadores, el diseño y administración de las prestaciones sociales, el desarrollo de la carrera profesional, etc.

- Propuestas de mejora: una vez analizadas cada una de las funciones y discutida su procedencia, se podrán realizar las propuestas de mejora que cada equipo estime de interés.

- Conclusiones: se dará una opinión general de la gestión realizada por el departamento de recursos humanos de la empresa analizada.

- Bibliografía: se indicará la relación de documentos empleada como apoyo para la elaboración del trabajo (esto es, libros, artículos de revistas, páginas web, etc.).

- Anexos: se adjuntará como mínimo la siguiente documentación:

1. El meta-análisis del equipo: se realizará un análisis del trabajo realizado por el equipo, los obstáculos con los que se ha encontrado, la forma de superarlos, las sinergias obtenidas y el rol desempeñado por cada uno de los miembros del equipo (véase el Apéndice 1).

2. El cuestionario elaborado para las entrevistas con el director o responsable de recursos humanos o, en su caso, gerente de la organización empresarial elegida.

3. La documentación original de las notas tomadas en el desarrollo de la entrevista.

4. Un CD que debe contener el audio de la entrevista (en formato mp3), la memoria del trabajo de campo (en formato Word o pdf), la presentación de la exposición pública (en Powerpoint) y cualquier otra documentación que el grupo considere de interés adjuntar. 
Finalmente, para concluir con la memoria los alumnos la revisan en una tercera y última tutoría ante los profesores.

\section{Evaluación de los trabajos y DE LAS PRESENTACIONES PÚBliCAS: RÚBRICAS DE EVALUACIÓN}

Con el fin de unificar y objetivar los criterios de evaluación del aprendizaje práctico de esta asignatura, se ha diseñado una rúbrica de evaluación de la memoria del trabajo de campo (véase el Apéndice 2), que sirve de guía a los alumnos para su implementación. En ella se especifican los criterios que se van a utilizar para evaluar dicho trabajo, así como la ponderación de cada uno de ellos. En concreto, se pondera con un 50\% el contenido y su correspondencia con el tema abordado, así como la adecuación del lenguaje utilizado. Un 20\% representa la relevancia de las propuestas de mejora y su concordancia con los contenidos expuestos. Con un $15 \%$ se ponderan las conclusiones que se alcanzan fruto de los resultados obtenidos en la exploración de campo. Un 10\% se reserva para evaluar la bibliografía utilizada y, finalmente, un 5\% lo constituye la evaluación formal de la portada y el índice.

Una vez realizada la memoria, los componentes de cada equipo deben realizar una exposición pública ante sus compañeros y profesores, que será evaluada por ambos, compañeros y profesores, de acuerdo con la rúbrica de evaluación de la exposición pública (véase el Apéndice 3). Dicha rúbrica también se ha diseñado con el mismo fin y, para ello, pondera por igual cada uno de los criterios de evaluación que utiliza. Esto es, la definición del trabajo, el uso de las nuevas tecnologías; la fluidez, claridad y organización de la exposición; la relevancia de los aspectos señalados, la coherencia y dominio de la materia; la originalidad y creatividad manifestadas; la adecuación del tiempo empleado en la exposición; y la seguridad y autocontrol demostrados durante la exposición pública.

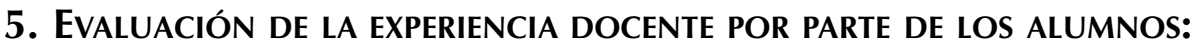 DESARROLLO DEL PLAN DE PRÁCTICAS Y ADQUISICIÓN DE COMPETENCIAS}

\subsection{Metodología del estudio}

Para evaluar el grado de satisfacción de los alumnos, tanto respecto al desarroIlo del plan de prácticas como en lo relativo a la valoración que hacen de las competencias transversales adquiridas a través del mismo, hemos aplicado un cuestionario a una muestra de 199 alumnos al finalizar dicha experiencia (Laffon y Pásaro, 2011; Cantonnet et al., 2013). Este cuestionario consta de 23 preguntas Likert (véase el Apéndice 4). Las trece primeras hacen referencia a la valoración del desarrollo del plan de prácticas, de acuerdo con el esquema propuesto previamente. Mientras que las 10 restantes valoran básicamente la adquisición de competencias transversales a través del plan de prácticas. La Tabla 1 recoge la ficha técnica del estudio realizado. 
Tabla 1. Ficha técnica del estudio

\begin{tabular}{|l|l|}
\hline Denominación & $\begin{array}{l}\text { Evaluación del grado de satisfacción respecto de la } \\
\text { experiencia docente }\end{array}$ \\
\hline Universo & $\begin{array}{l}334 \text { alumnos de la asignatura de Comportamiento } \\
\text { Organizativo de la UR }\end{array}$ \\
\hline Unidad de análisis & Cada uno de los alumnos \\
\hline Técnica de recogida de datos & $\begin{array}{l}\text { Cuestionario Likert realizado en clase al finalizar la } \\
\text { experiencia docente }\end{array}$ \\
\hline Tamaño de la muestra & 199 alumnos que contestaron el cuestionario \\
\hline Error muestral & $\pm 4,42 \%$ \\
\hline Nivel de confianza & $95 \%$ \\
\hline Fecha del trabajo de campo & Segunda semana de mayo de 2012 \\
\hline Técnicas de análisis empleadas & Análisis descriptivo e inferencial \\
\hline
\end{tabular}

\subsection{Resultados del desarrollo del plan de prácticas}

Los alumnos han valorado muy satisfactoriamente su participación en dicha experiencia a tenor de los resultados recogidos en la Figura 2, que muestran que la valoración 4 (de una escala de 1 a 5) es la que presenta una mayor frecuencia para todas las preguntas, excepto para las preguntas segunda, tercera, octava y novena que lo son para la valoración 3. No obstante, el test paramétrico de la media y el test no paramétrico de la mediana nos confirman que la valoración que los alumnos han otorgado a cada una de las 13 preguntas está muy por encima de tres (véase Tabla 2). Finalmente, la valoración general del plan de prácticas, sintetizada en la pregunta decimotercera, es muy positiva y está en consonancia con las demás valoraciones parciales, recogidas en el resto de preguntas.

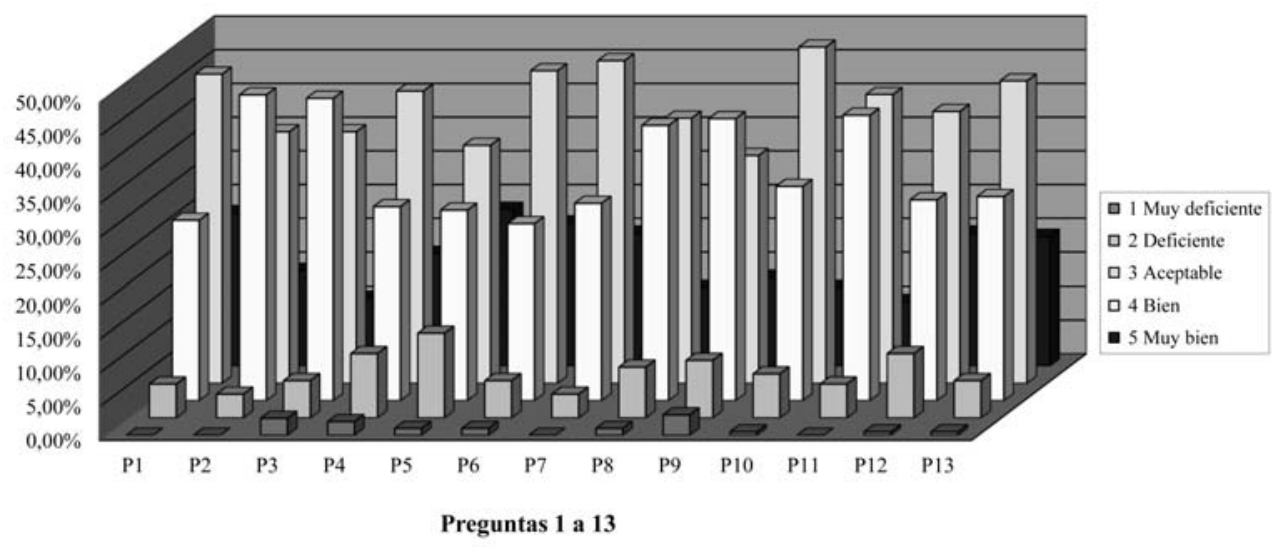

Figura 2. Histograma de frecuencias de la valoración del desarrollo del plan de prácticas 
Tabla 2. Contrastes de la media y la mediana para la valoración del desarrollo del plan de práctica

\begin{tabular}{|l|c|c|c|c|c|c|c|c|c|c|c|c|c|}
\hline Estadístico & P1 & P2 & P3 & P4 & P5 & P6 & P7 & P8 & P9 & P10 & P11 & P12 & P13 \\
\hline Media & 3,86 & 3,62 & 3,47 & 3,63 & 3,67 & 3,81 & 3,83 & 3,53 & 3,45 & 3,65 & 3,57 & 3,69 & 3,76 \\
\hline Mediana & 3,86 & 3,62 & 3,47 & 3,63 & 3,67 & 3,81 & 3,83 & 3,53 & 3,45 & 3,65 & 3,57 & 3,69 & 3,76 \\
\hline t-student & 14,73 & 11,34 & 7,80 & 9,44 & 9,43 & 13,17 & 15,15 & 8,93 & 6,86 & 11,69 & 10,95 & 10,69 & 12,82 \\
\hline Chi-cuadrado & 79,78 & 45,35 & 30,57 & 46,31 & 39,80 & 73,57 & 81,05 & 35,46 & 24,62 & 58,61 & 44,41 & 49,26 & 66,46 \\
\hline
\end{tabular}

Nota: Todos los valores de la t-student y Chi-cuadrado son significativos al $1 \%$

\subsection{Resultados sobre la adquisición de competencias transversales}

De nuevo los alumnos han valorado muy satisfactoriamente la adquisición de competencias transversales a través de dicha experiencia, dado que, tal y como revela la Figura 3, la valoración 4 (de una escala de 1 a 5) es la que presenta una mayor frecuencia para todas las preguntas. Además, el test paramétrico de la media y el test no paramétrico de la mediana nos confirman que la valoración que los alumnos han otorgado está muy por encima de tres para cada una de las preguntas (véase Tabla 3). También aquí la valoración general, sintetizada en la pregunta vigésima tercera, es muy positiva y permite afirmar que el plan de prácticas desarrollado consigue mejorar ampliamente la formación en competencias transversales del alumnado.

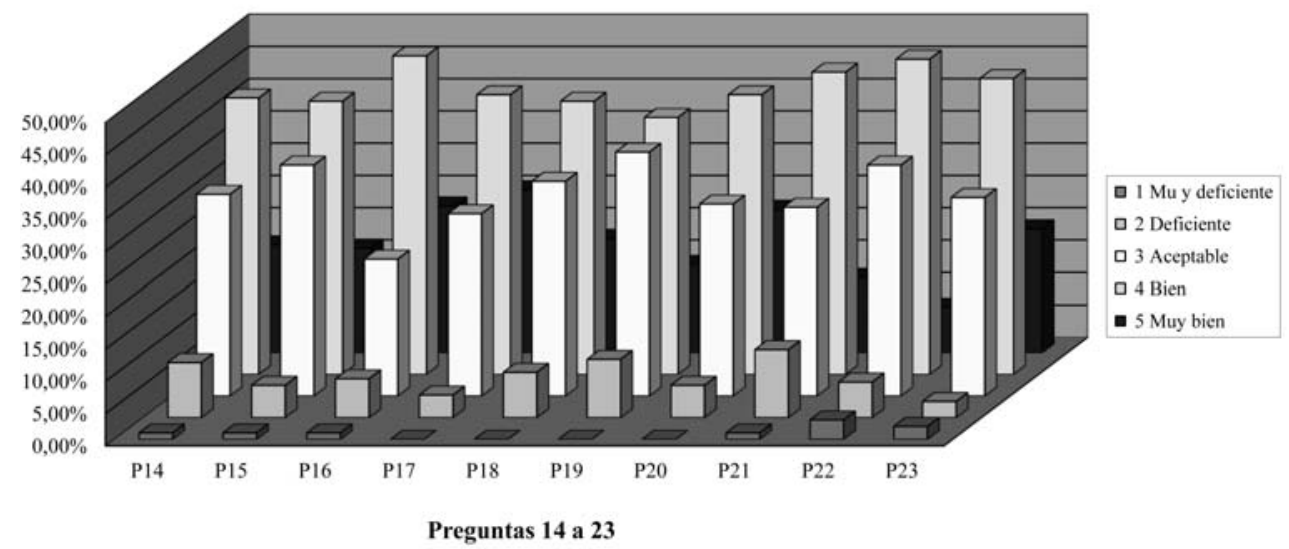

Figura 3. Histograma de frecuencias de la valoración de las competencias transversales adquiridas 
Tabla 3. Contrastes de la media y la mediana para la valoración de las competencias transversales adquiridas

\begin{tabular}{|l|c|c|c|c|c|c|c|c|c|c|}
\hline Estadístico & P14 & P15 & P16 & P17 & P18 & P19 & P20 & P21 & P22 & P23 \\
\hline Media & 3,65 & 3,67 & 3,86 & 3,90 & 3,70 & 3,58 & 3,82 & 3,58 & 3,51 & 3,77 \\
\hline Mediana & 3,65 & 3,67 & 3,86 & 3,90 & 3,70 & 3,58 & 3,82 & 3,58 & 3,51 & 3,77 \\
\hline t-student & 10,35 & 11,31 & 14,04 & 15,54 & 11,82 & 9,75 & 13,98 & 9,39 & 8,73 & 12,76 \\
\hline Chi-cuadrado & 49,25 & 54,35 & 83,62 & 83,62 & 55,40 & 38,91 & 72,36 & 43,48 & 44,40 & 72,36 \\
\hline
\end{tabular}

Nota: Todos los valores de la t-student y Chi-cuadrado son significativos al $1 \%$

\section{SUMARIO Y CONCLUSIONES}

El desarrollo de esta experiencia ha supuesto un reto importante, tanto para los alumnos como para los profesores implicados, al requerir de nuevas formas de abordar el proceso de aprendizaje para los alumnos y de enseñanza para los profesores. La experiencia ha resultado muy satisfactoria para todos a tenor de lo que hemos venido observando a través del desarrollo de la misma (comentarios de los alumnos, evolución de los grupos de trabajo, reuniones con los equipos de trabajo, exposiciones públicas, etc.) y de la valoración que los alumnos le han otorgado.

El contacto directo con la realidad laboral, mediante el desarrollo del plan de prácticas propuesto, ha supuesto para la mayoría de los alumnos una novedad impactante pero, a su vez, tremendamente enriquecedora, como así se desprende de la valoración general que los alumnos han otorgado a dicha experiencia vivida. Sus comentarios a lo largo del curso abundan en este sentido. En particular, los relativos al esfuerzo que les supone encontrar una organización empresarial que les abra sus puertas, la coordinación y distribución de tareas que necesariamente deben establecer entre los miembros del equipo, la relación que tienen que entablar con los profesionales de dicha organización explorada, el análisis y discusión en grupo de los datos obtenidos, la creatividad requerida a la hora de proponer alternativas de mejora a la organización, y la ansiedad que les supone, a unos más que a otros, la presentación y defensa del trabajo ante sus compañeros y profesores. Además, todo parece indicar que el esfuerzo realizado lo dan por bien empleado, ya que se consideran más competentes profesionalmente y con mayor confianza en sí mismos, como así es posible atestiguarlo a través de la evolución de cada grupo de trabajo desde la primera tutoría de supervisión hasta la defensa pública del trabajo de campo. Esta opinión positiva se confirma a través de la valoración general que los alumnos han otorgado a la adquisición de competencias transversales, al considerar que la experiencia vivida a través del plan de prácticas consigue, en términos generales, mejorar la formación del alumno.

La valoración que hacemos los profesores implicados en esta experiencia es muy positiva, ya que ha aumentado y mejorado la planificación y coordinación entre nosotros (especialmente la unificación y objetivación de los criterio de evaluación). A su vez, la atención personalizada al alumno, en grupo pequeño, ha propiciado la evaluación continua, y, finalmente, los alumnos han tenido la oportunidad de expe- 
rimentar y poner en práctica los contenidos teóricos de la asignatura. En definitiva, creemos que supone una innovación docente muy enriquecedora para los alumnos en la línea con las nuevas exigencias demandadas por el nuevo Espacio Europeo de Educación Superior.

\section{REFERENCIAS BIBLIOGRÁFICAS}

ASTORECA, J. S. (2012). Experiencia docente en el marco de Dirección de Empresa. UPO INNOVA: Revista de Innovación Docente, 1, 49-53.

BÉJAR, M. y MERINO, L. (2012). Una visión práctica de la implantación del EEES: metodología docente y sistema de evaluación. UPO INNOVA: Revista de Innovación Docente, 1, 64-85.

CANTONNET, M. L.; BERBEGAL, J. y ALDASORO, J. C. (2013). Análisis de la adaptación al Espacio Europeo de Educación Superior (EEES) a través de las encuestas de satisfacción del alumnado. El caso de la asignatura de Seguridad y Prevención de Ingeniería de la Edificación de la Universidad del País Vasco (España). Tendencias Pedagógicas, 21, 119-132.

FLÓREZ, R. y ALBELDA, E. (2012). Una experiencia de trabajo en equipo con soporte documental para la formación de competencias en Contabilidad Financiera. Revista de Educación en Contabilidad, Finanzas y Administración de Empresas, 3, 67-94.

GALLEGO, M. J. y RAPOSO-RIVAS, M. (2014). Compromiso del estudiante y percepción del proceso evaluador basado en rúbricas. Revista de Docencia Universitaria, 12 (1), 197-215.

GARCÍA-ROS, R. (2011). Análisis y validación de una rúbrica para evaluar habilidades de presentación oral en contextos universitarios. Electronic Journal of Research in Educational Psychology, 9 (3), 1043-1062.

GARCÍA-ROS, R. y IQDOCENT - Grupo de Innovación Educativa de la Universitat de Valencia, Grupo de Trabajo del Practicum de Psicología (2012). Diseño y utilización de rúbricas en la enseñanza universitaria: Una aplicación en la titulación de Psicología. Electronic Journal of Research in Educational Psychology, 10 (3), 1477-1492.

GUTIÉRREZ, S. y DE PABLOS, C. (2010). Análisis y evaluación de la gestión por competencias en el ámbito empresarial y su aplicación a la universidad. Revista Complutense de Educación, 21 (2), 323-343.

ION, G. y CANO, E. (2012). La formación del profesorado universitario para la implementación de la evaluación por competencias. Educación XXI, 15 (2), 249-270.

JENARO, C.; FLORES, N.; POY, R.; GONZÁLEZ-GIL, F. y MARTÍN-PASTOR, E. (2013). Metodologías docentes en la educación superior: percepciones del profesorado sobre su importancia y uso. Revista de Enseñanza Universitaria, 39, 1-16.

LAFFON, B. y PÁSARO, E. (2011). Adaptación de una Asignatura de Logopedia al Espacio Europeo de Educación Superior, EEES: Percepción de los Estudiantes. Formación Universitaria, 4 (5), 13-20. 
MARTÍNEZ-FIGUEIRA, E.; TELLADO-GONZÁLEZ, F. y RAPOSO-RIVAS, M. (2013). La rúbrica como instrumento para la autoevaluación: un estudio piloto. Revista de Docencia Universitaria, 11 (2), 373-390.

PALOMARES, A. (2011). El modelo docente universitario y el uso de nuevas metodologías en la enseñanza, aprendizaje y evaluación. Revista de Educación, 355, 591-604.

PANADERO, E. y JONSSON, A. (2013). The use of scoring rubrics for formative assessment purposes revisited: A review. Educational Research Review, 9, 129-144.

SAYAGO, A.; HERNANZ, D.; GALLO, V. y BELTRÁN, R. (2011). Adaptación de las prácticas de Análisis Instrumental al Espacio de Convergencia Europeo. Elaboración de Material Didáctico. Formación Universitaria, 4 (1), 19-24. 


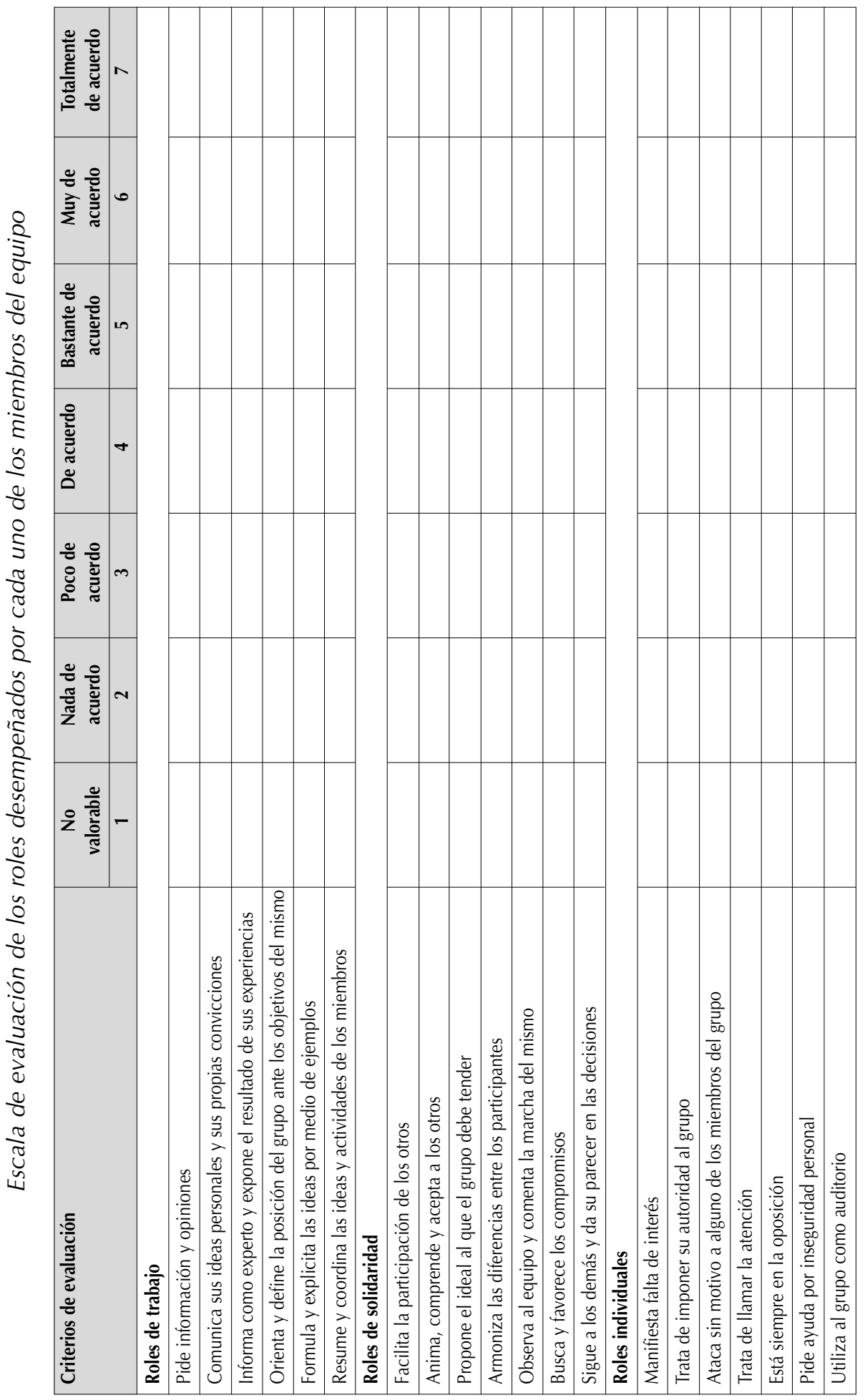




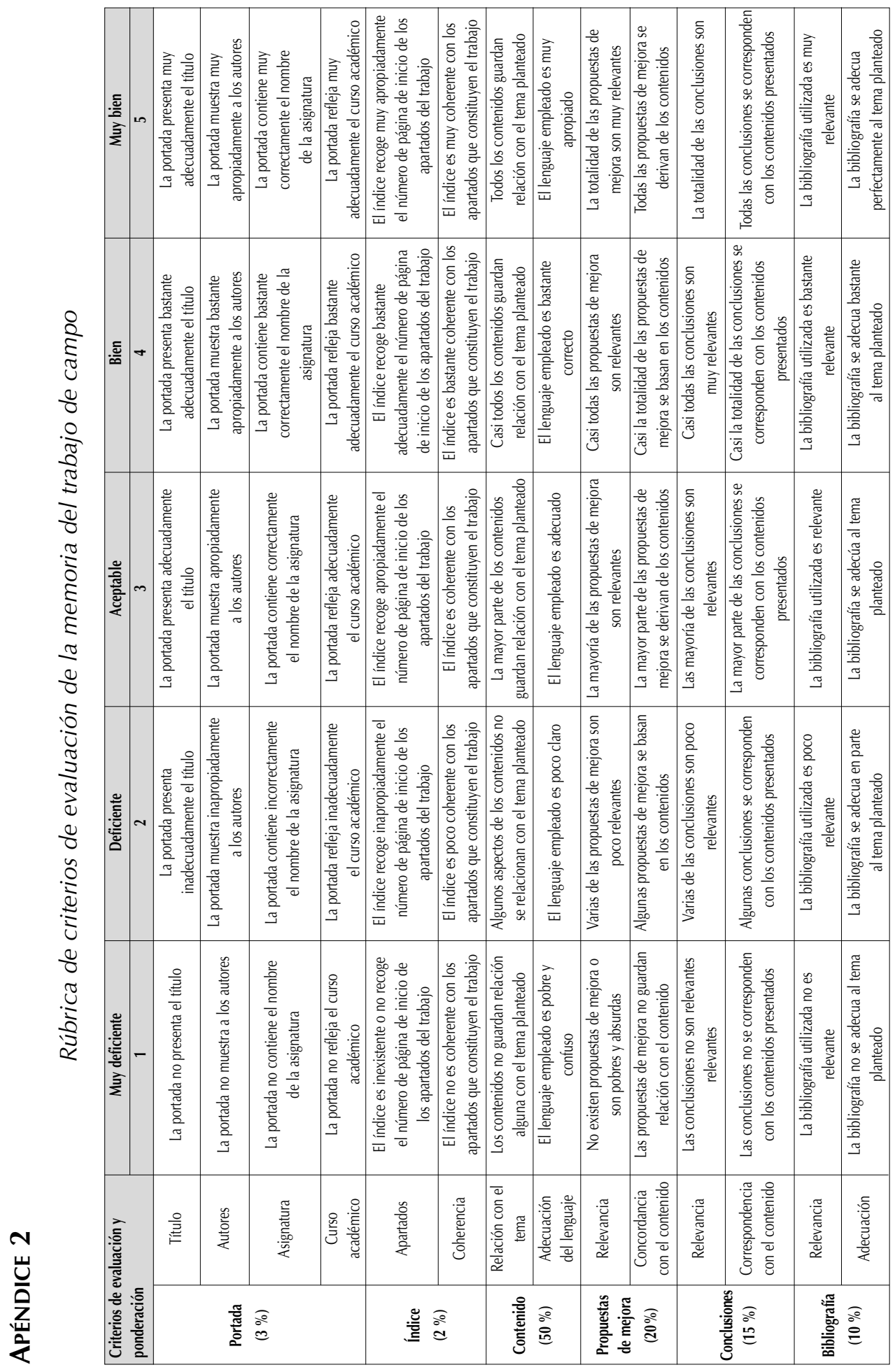




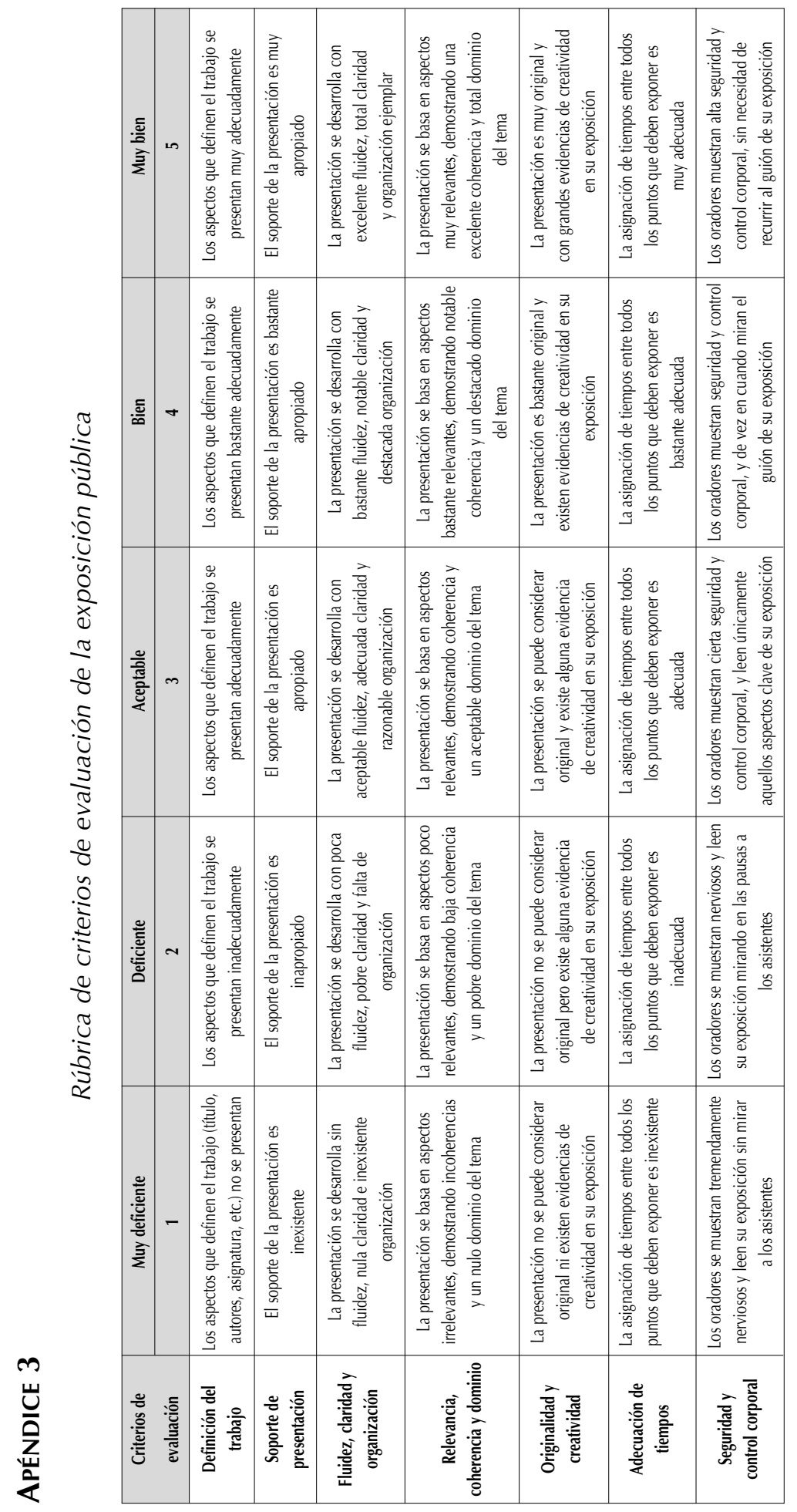




\title{
APÉNDICE 4
}

\author{
Cuestionario para la valoración que los estudiantes otorgan a su participación \\ en el plan de prácticas
}

\begin{tabular}{|c|c|c|c|c|c|c|c|c|}
\hline \multicolumn{9}{|c|}{ Responda a los ítems en orden creciente, según este modelo de valoración } \\
\hline $\begin{array}{l}\text { Muy deficiente } \\
1\end{array}$ & $\begin{array}{l}\text { Deficiente } \\
\quad 2\end{array}$ & $\begin{array}{c}\text { Aceptable } \\
3\end{array}$ & $\begin{array}{c}\text { Bien } \\
4\end{array}$ & \multicolumn{5}{|c|}{$\begin{array}{l}\text { Muy bien } \\
5\end{array}$} \\
\hline \multicolumn{4}{|l|}{ Ítems a valorar } & \multicolumn{5}{|c|}{ Indicar con una X } \\
\hline & & & & 1 & 2 & 3 & 4 & \\
\hline \multicolumn{4}{|c|}{ Valoración del desarrollo del plan de prácticas } & & & & & \\
\hline \multicolumn{4}{|c|}{ 1. Los objetivos del plan de prácticas fueron claramente definidos por los profesores } & & & & & \\
\hline \multicolumn{4}{|c|}{$\begin{array}{l}\text { 2. La descripción y discusión del trabajo preliminar (proyecto) ante los profesores clarificó } \\
\text { los pormenores del trabajo posterior a desarrollar }\end{array}$} & & & & & \\
\hline \multicolumn{4}{|c|}{$\begin{array}{l}\text { 3. La búsqueda y selección de bibliografía, así como su posterior estudio, propició el } \\
\text { aprendizaje autónomo }\end{array}$} & & & & & \\
\hline \multicolumn{4}{|c|}{ 4. La supervisión del cuestionario por parte de los profesores enriqueció el cuestionario final } & & & & & \\
\hline \multicolumn{4}{|c|}{$\begin{array}{l}\text { 5. Las visitas a la organización empresarial y las entrevistas con sus directivos proporcionaron } \\
\text { los datos necesarios para su posterior análisis y discusión }\end{array}$} & & & & & \\
\hline \multicolumn{4}{|c|}{$\begin{array}{l}\text { 6. Este contacto con las organizaciones empresariales y sus directivos contribuyó al } \\
\text { acercamiento de la realidad empresarial al aula }\end{array}$} & & & & & \\
\hline \multicolumn{4}{|c|}{$\begin{array}{l}\text { 7. El análisis de los datos obtenidos y su posterior discusión en el seno del equipo de trabajo } \\
\text { permitió un aprendizaje reflexivo y crítico }\end{array}$} & & & & & \\
\hline \multicolumn{4}{|c|}{$\begin{array}{l}\text { 8. La rúbrica de los criterios de evaluación de la memoria del trabajo facilitó la elaboración } \\
\text { de la memoria }\end{array}$} & & & & & \\
\hline \multicolumn{4}{|c|}{ 9. La memoria final mejoró tras la revisión por parte de los profesores } & & & & & \\
\hline \multicolumn{4}{|c|}{$\begin{array}{l}\text { 10. La rúbrica de los criterios de evaluación de la exposición y defensa del trabajo favoreció } \\
\text { la preparación de su exposición pública }\end{array}$} & & & & & \\
\hline \multicolumn{4}{|c|}{$\begin{array}{l}\text { 11. Las rúbricas de los criterios de valuación (de la memoria y de la exposición y defensa) } \\
\text { unificaron criterios y aportaron objetividad al proceso de evaluación }\end{array}$} & & & & & \\
\hline \multicolumn{4}{|c|}{$\begin{array}{l}\text { 12. La planificación y ejecución del trabajo del plan de prácticas estuvo presidida por una } \\
\text { cultura colaborativa de integración de los miembros del equipo de trabajo }\end{array}$} & & & & & \\
\hline \multicolumn{4}{|c|}{ 13. Qué valoración general le otorgas a la experiencia vivida a través del plan de prácticas } & & & & & \\
\hline \multicolumn{4}{|c|}{ Valoración de las competencias transversales adquiridas a través del plan de prácticas } & & & & & \\
\hline \multicolumn{4}{|c|}{ 14. Implica y motiva a los alumnos en el desarrollo práctico de la asignatura } & & & & & \\
\hline \multicolumn{4}{|c|}{ 15. Favorece el trabajo autónomo del alumno y, por tanto, su autoaprendizaje } & & & & & \\
\hline \multicolumn{4}{|c|}{ 16. Pone en práctica actitudes colaborativas entre alumnos y la cultura del trabajo en equipo } & & & & & \\
\hline \multicolumn{4}{|c|}{$\begin{array}{l}\text { 17. Consigue que el alumno se aproxime a la realidad de las competencias profesionales que } \\
\text { la sociedad, en su conjunto, y el mundo empresarial, en particular, le van a exigir }\end{array}$} & & & & & \\
\hline \multicolumn{4}{|c|}{ 18. Propicia la reflexión y valoración crítica del alumno } & & & & & \\
\hline \multicolumn{4}{|c|}{ 19. Enriquece la comunicación escrita del alumno (elaboración de la memoria) } & & & & & \\
\hline \multicolumn{4}{|c|}{ 20. Desarrolla la comunicación oral del alumno (exposición pública de la memoria) } & & & & & \\
\hline \multicolumn{4}{|c|}{$\begin{array}{l}\text { 21. Contribuye a la utilización de las nuevas Tecnologías de la Información y Comunicación } \\
\text { (TICs) }\end{array}$} & & & & & \\
\hline \multicolumn{4}{|c|}{ 22. Proporciona un sistema de evaluación acorde con las competencias a evaluar } & & & & & \\
\hline \multicolumn{4}{|c|}{ 23. Consigue, en términos generales, mejorar la formación del alumno } & & & & & \\
\hline
\end{tabular}

